\title{
An excursion into classical nonlinear acoustics
}

\author{
Niko Sauer \\ Department of Mathematics and Applied Mathematics, University of Pretoria, Pretoria 0002, South Africa
}

\section{A R T I C L E I N F O}

\section{Article history:}

Received 2 November 2009

Accepted 15 February 2010

Available online $\mathrm{xxxx}$

Communictaed by K.R. Rajagopal

\section{Keywords:}

Nonlinear acoustics

Shock discontinuity

Dynamic piston problem

\begin{abstract}
A B S T R A C T
This paper is concerned with the derivation of the partial differential equations that govern the propagation of sonic disturbances in an ideal gas under isentropic conditions. The result is a quasilinear hyperbolic system of first order equations and an inequality constraint. The speed of propagation is pressure dependent. It is shown how to deal with the equations and the constraint and how to calculate characteristics and solutions. It is also shown that shock discontinuities can develop which distinguishes the equations from the traditional linear wave equation.
\end{abstract}

(c) 2010 Published by Elsevier Ltd.

\section{Introduction}

The term 'nonlinear acoustics' at present seems to refer to the propagation of acoustic disturbances in nonlinear materials (see e.g. [14]). Classical acoustics is by and large the study of disturbances in non-viscous gases. In both fields variants of the wave equation are either in the background or quite dominant. In classical acoustics the wave equation is simply the linear one. Since the term 'wave' essentially refers to solutions of the linear wave equation, the term 'sound wave' became firmly entrenched.

An early attempt at finding the form of a differential equation which will describe the propagation of acoustic disturbances in a gas (air) came from Earnshaw [4,5] who contemplated the possibility of the formation of bores (see e.g. [16]) when a piston is pushed into a pipe. The heuristic argumentation lead to the intuition that the propagation speed of the disturbance must be pressure dependent. In his venerated treatises on the theory of sound (of late two volumes in a single cover) Lord Rayleigh attempts to find the precise form of Earnshaw's equation [17, Vol. II, p. $37 \mathrm{ff}$ ] but soon resorts to the linear wave equation.

Systematic derivations of the equation of classical acoustics only seem to have been presented after the time of Rayleigh. For the one-dimensional case one of the first can be found in Coulson [1] where a concerned effort was made to identify the approximation that results in the linear wave equation. Another derivation can be found in Serrin [15, p. 180] where the Euler equations for a frictionless gas and the principle of conservation of mass are used. It is of interest to note that in [1] the Lagrangian description of the motion of a continuum is used implicitly.

A serious shortcoming of the linear wave equation as basis for classical acoustics is that the formation of shock discontinuities cannot be explained. When the Euler equations for the motion of a non-viscous gas together with the conservation of mass equation (in the Eulerian description) are used as a quasilinear system of first order partial differential equation, the phenomenon can occur. The famous treatise of Courant-Friedrichs [2] deals with this in a very lucid way. This could be the basis for classical acoustics.

The first aim of this paper is to derive, in the spirit of present-day rational mechanics, a system of nonlinear partial differential equations that is at the core of classical acoustics. Since the equations are not linear, this is somewhat in harmony

E-mail address: nikos@friedrichs.up.ac.za 
with the concept of nonlinear acoustics. The second aim is to analyze the special case of one-dimensional motion in order to compare the consequences with the well-known behaviour derived from linear wave motion. The underlying framework will be the Lagrangian description in which individual particles and volumes consisting of the same particles are followed in time. This approach is of fundamental importance when thermodynamics is brought into consideration. It is also convenient in the analysis of sound systems because the domains considered there will not be time-dependent. Section 2 is devoted to setting up notation and the basic notions for this approach.

In Sections 3 and 4 the physical principles of conservation of mass and balance of linear momentum are set out and combined in a manner suitable for our exposition. Section 5 is a small diversion into classical thermo-statics and the traditional theory of sound. Here we introduce the notion of compression in a static/global sense. It is defined as a ratio of volumes which could be viewed as purely geometrical but soon joins with the relevant physical concepts. This leads to a relationship between compression and pressure which may be very fruitful in the study of acoustical phenomena.

Section 6 is devoted to localizing the notion of compression by letting the ratio of volumes consisting of the same gas particles shrink to a point. Combined with the equation that represents the conservation of mass an exact analogue of a static expression from the previous section is obtained. It also leads to a localized version of the compression-pressure relation which we put forward as the basic acoustic postulate. Once this is done the fundamental dynamic equations of nonlinear acoustics appear as a system of first order nonlinear partial differential equations which shows that the speed of propagation of acoustic disturbances is pressure dependent. Such a dependence was alluded to in [4,5]. The scaled version of these equations (Section 7) make them much clearer and shows that the acoustic hypothesis resembles the classic Boyle-Marriott law. Another consequence of the arguments used to derive the acoustic equations is an inequality constraint on the pressure which represents the fact that it is impossible to compress a body of gas until there is no volume.

The simplest case is that of one-dimensional motion in which the system of equations reduces to two equations for two unknowns, velocity and pressure (Section 8). This is followed in Section 9 with a 'plausibility' argument to obtain the wellknown linear system of equations. It is seen immediately that the linear system cannot possibly yield approximate solutions for the nonlinear system. One reason is that the linear system is symmetric in velocity and pressure while the nonlinear system is not. Moreover, the constraint mentioned above is not seen in the linearized equations while it is crucial in the nonlinear system. In Section 10 the constraint is utilized to transform the one-dimensional equations into a symmetric hyperbolic system which exhibits the true nonlinear character of the problem at hand.

The remaining sections of the paper analyzes the nonlinear system on the (spatial) real line for the purpose of demonstrating its response to various inputs. The method of characteristics is used and this requires a numerical method which is suggested by obtaining from the differential equations of the characteristics a crucial integral equation. It is shown that shock discontinuities can occur and this manifests in the results of numerical computations. Of significance is the observation that the roles of initial velocity and pressure inputs cannot be exchanged. It is also shown that violation of the constraint leads to a result similar to the sonic boom. This is further evidence that the linearization is by no means an approximation of anything outside of virtual reality. Finally it is shown how the well-known piston problem should be handled, thus vindicating Earshaw's early thinking.

\section{The Lagrange approach in hydrodynamics}

In this approach, which is quite suitable for the study of sound propagation, we follow the motion of individual fluid 'particles' in contrast to the field theoretical (Euler) approach in which the state of the fluid motion at arbitrary fixed points $x=\left(x_{1}, x_{2}, x_{3}\right)$ in three-dimensional Euclidean space are considered. For this purpose we use a reference configuration of fluid particles (the rest state, for example) $\mathscr{B} \subset \mathrm{R}^{3}$ which does not depend on time $(t)$. The particles in the fluid are identified by their position $x \in \mathscr{B}$. One could think of $x$ as the 'name' of the particle. The motion of the fluid is described by a function $\psi(\cdot, t): x \in \mathscr{B} \mapsto X(t):=\psi(x, t)$ with $X(t)$ the position of $x$ at time $t$. It is natural to assume that $\psi$ is one-to-one, for otherwise two distinct particles could end up in the same position at some time $t$ and names would not be unique.

For the fluid to have 'bulk' at all, we assume that the reference configuration $\mathscr{B}$ is an open subset of $\mathrm{R}^{3}$ and we shall consider only very smooth motions. That is, we assume that $\psi$ and its inverse $\psi^{-1}$ have continuous derivatives with respect to all variables. The derivatives will be denoted by $\partial_{t}:=\frac{\partial}{\partial t}$ and $\partial_{k}:=\frac{\partial}{\partial x_{k}}$. It is immediately seen, by virtue of the fact that $\psi(x, t)=\left(\psi_{1}(x, t), \psi_{2}(x, t), \psi_{3}(x, t)\right)$, that $\partial_{t} \psi(x, t)$ is a vector quantity.

Our first objective is to find, on the basis of classical physics, a system of partial differential equations that will enable us to determine the motion $\psi$. These will be discussed in the following three sections. We prepare for this by introducing the notion of the gradient of 3-vector fields. Let $f(x)=\left(f_{1}(x), f_{2}(x), f_{3}(x)\right)$ be a differentiable 3-vector field defined for $x \in \Omega \subset \mathrm{R}^{3}$, with $\Omega$ an open set. Then the gradient $L$ of $f$ is defined as the matrix function with components $L_{x, i j}(f)(x)=\partial_{j} f_{i}(x)$. The transpose matrix function $L_{x}^{T} f$ is defined as the transpose of $L_{x} f(x)$. The motion $\psi(\cdot, t)$ is simply an invertible transformation from one coordinate system $\{x\}$ to another $\{X\}$ with Jacobian $J(x, t):=\operatorname{det}\left[L_{x} \psi(x, t)\right]$. The Jacobian can never be zero and by suitable choices of the directions of coordinate axes, it may be assumed that $J(x, t)$ is positive.

If an $n \times n$ matrix $A$ is invertible, its transpose $A^{T}$ is also invertible and $\left[A^{T}\right]^{-1}=\left[A^{-1}\right]^{T}=: A^{-T}$. We shall be in need of this abusive notation. Suppose that $F(X, t)$ is a scalar field defined on the configuration of fluid particles at time $t$. Let $f(x, t):=F(\psi(x, t), t)$. Then it is seen from the chain rule that $\nabla_{x} f(x, t)=L_{x}^{T} \psi(x, t) L_{X} F(X, t)$. We therefore have

$$
\nabla_{X} F(X, t)=L_{x} \psi(x, t)^{-T} L_{x} f(x, t) .
$$


If $A$ and $B$ are $n \times n$ matrices with components $a_{i j}$ and $b_{i j}$ we use the notation $A: B:=\sum_{i j=1}^{n} a_{i j} b_{i j}$. If $F$ and $f$ above are vector fields related by the transformation $\psi$ we have for the divergence in $X$-coordinates

$$
\nabla_{X} \cdot F(X, t)=L_{x} \psi(x, t)^{-T}: L_{x} f(x, t) .
$$

\section{Conservation of mass}

The principle of conservation of mass states that the mass of an arbitrary collection of particles is constant as it moves along in time. To make this mathematically precise, let $\mathscr{G}$ be an open subset of $\mathscr{B}$ and let $\mathscr{G}(t):=\psi(\cdot, t)[\mathscr{G}]$ be its configuration at time $t$. Then the mass of $\mathscr{G}$ is the same as the mass of $\mathscr{G}(t)$.

To make the principle mathematically workable we use the notion of mass density. This is a function from which the mass of a given configuration of fluid particles may be computed. Since the fluid is compressible, the mass density (mass per unit of volume) may change. Let $\rho(x)$ be the density function in the reference configuration and $\sigma(X, t)$ the density function in the configuration at time $t$. The conservation of mass then simply states that

$$
\int_{\mathscr{G}} \rho(x) d x=\int_{\mathscr{G}(t)} \sigma(X, t) d X .
$$

The integrals in this expression are volume integrals and the volume measures such as $d x$ should be thought of as being $d x=d x_{1} d x_{2} d x_{3}$. The right of (3.1) may be transformed into an integral over the reference volume $\mathscr{G}$ by letting $X=\psi(x, t)$. By the familiar rule we then obtain

$$
\int_{\mathscr{G}} \rho(x) d x=\int_{\mathscr{G}} \sigma(\psi(x, t), t) J(x, t) d x .
$$

Since the expression (3.2) holds for arbitrary open subsets of $\mathscr{B}$, a standard theorem states that the integrands must be equal. We therefore have

$$
\rho(x)=J(x, t) \sigma(\psi(x, t), t),
$$

which is the principle of conservation of mass expressed in terms of mass densities.

\section{Balance of linear momentum}

To formulate the principle of balance of linear momentum it is necessary to discuss the concept of velocity first. The velocity of particle $x$ at time $t$ is defined as $v(x, t):=d X / d t=\psi_{t}(x, t)$. The velocity observed at $X$ at time $t$ is denoted by $V(X, t)$. This, of course, is the same as $v(x, t)$ if $X=\psi(x, t)$.

Since we deal with an ideal gas (no viscosity) the internal force observed at $X$ at time $t$ is due to pressure only. We assume that there are no external forces. If $\mathscr{G}$ is an arbitrary open set of the reference configuration and $\mathscr{G}(t)=\psi(\cdot, t)[\mathscr{G}]$ its image at time $t$, the principle of balance of linear momentum may be expressed by

$$
\frac{d}{d t} \int_{\mathscr{G}(t)} \sigma(X, t) V(X, t) d X=-\int_{\mathscr{O G}(t)} P(X, t) n(X) d s=-\int_{\mathscr{G}(t)} \nabla_{X} P(X, t) d X
$$

Here $P(X, t)$ is the pressure observed at $X$ at time $t$ and $n(X)$ the unit exterior normal at $X$ to the surface $\partial \mathscr{G}(t)$. The minus sign on the right is due to the use of the exterior normal in the expression. The final expression in (4.1) is obtained from the divergence theorem.

Balance of linear momentum can under certain assumptions be expressed as a partial differential equation. For this we transform the expression (4.1) to the reference coordinates $(x)$ by $X=\psi(x, t)$ to obtain

$$
\frac{d}{d t} \int_{\mathscr{G}} \rho(x) v(x, t) d x=\frac{d}{d t} \int_{\mathscr{G}} J(x, t) \sigma(\psi(x, t), t) v(x, t) d x=-\int_{\mathscr{G}} J(x, t) L_{x} \psi(x, t)^{-T} \nabla_{x} p(x, t)
$$

with $p(x, t):=P(\psi(x, t), t)$. We have used conservation of mass (3.3) as well as the expression (2.1) to obtain this equation. We have assumed differentiability of the pressure to get this far. If, in addition, we assume that the time derivative and the integral in (4.2) can be interchanged and that $v$ has an integrable time derivative, we obtain

$$
\int_{\mathscr{G}}\left[\rho(x) v_{t}(x, t)+J(x, t) L_{x} \psi(x, t)^{-T} \nabla_{x} p(x, t)\right] d x=0 .
$$

Since $\mathscr{G}$ is an arbitrary open subset of $\mathscr{B}$ it follows that the integrand in (4.3) is zero. Thus we obtain our first dynamic equation

$$
\rho(x) v_{t}(x, t)+J(x, t) L_{x} \psi(x, t)^{-T} \nabla_{x} p(x, t)=0 .
$$




\section{Thermo-statics}

The system (4.4) of partial differential equations represents three equations for four unknown functions. We therefore need another equation which will be motivated by the use of thermodynamical considerations. In terms of classical thermodynamics (thermo-statics) these are as follows:

Consider a closed container of compressible gas in a 'reference state' where the volume of gas is $V_{0}$ and its density is $\rho$. This is compared to another state in which the volume is $V$ and the density is $\sigma$. The compression of the gas (relative to the reference state) is defined as

$$
r=\frac{V-V_{0}}{V_{0}}=\frac{V}{V_{0}}-1
$$

From the conservation of mass which proclaims that in both states the mass of gas in the container is the same, we conclude that

$$
\frac{V}{V_{0}}=\frac{\rho}{\sigma}=1+r,
$$

having used (5.1). The expression (5.2) is often used to define the notion of 'condensation'.

The standard thermo-static assumption is that the density of the gas in the state $\langle V, \sigma\rangle$ is determined by the pressure $p$ in that state. That is

$$
\sigma=f(p) \text {. }
$$

The static acoustic assumption is that for an isentropic gas the rate of change of density with pressure is constant and positive:

$$
\frac{d \sigma}{d p}=f^{\prime}(p)=c^{-2} .
$$

The constant $c$ will be referred to as the static sound speed.

From (5.2) and (5.3) it is seen that the compression is determined by pressure. Indeed, $\rho /(1+r)=f(p)$ and it follows from (5.4) that $-(1+r)^{-2} d r / d p=1 / \rho c^{2}$. This differential equation under the initial condition $r\left(p_{0}\right)=0$ can readily be integrated to obtain

$$
\frac{1}{1+r}=1+\frac{p-p_{0}}{\rho c^{2}}
$$

We need to remark that the relation (5.3) is usually stated differently in the sense that pressure is assumed to be a function of density. The formulation above is in accordance with the thinking of K.R. Rajagopal that change of pressure results in change of density. These deliberations have far-reaching consequences in continuum mechanics when constitutive relations are not invertible (see e.g. [13]).

\section{The dynamics of compression}

Since we use the Lagrangian description of continuum motion, the notion of compression as set out above can be localized. The compression $r(x, t)$ experienced by particle $x$ at time $t$ is defined as

$$
r(x, t):=\lim _{|\mathscr{G}| \rightarrow 0} \frac{|\mathscr{G}(t)|-|\mathscr{G}|}{|\mathscr{G}|} ; \quad x \in \mathscr{G} .
$$

Here the absolute value denotes volume. Since $\mathscr{G}$ and $\mathscr{G}(t)$ are representations of the same material, this definition is in line with the considerations of the previous section. The expression (6.1) can be evaluated by transforming the integral which defines the volume of $\mathscr{G}(t)$ to an integral over $\mathscr{G}$ (as we have done before). This leads to

$$
r(x, t)=J(x, t)-1 .
$$

We see that the compression $r$ only involves the kinematics of the motion $\psi$. It is, however related to the physical principle of conservation of mass as expressed in (3.3). By eliminating the Jacobian $J$ from both expressions we obtain

$$
r(x, t)=\frac{\rho(x)}{\sigma(\psi(x, t), t)}-1 .
$$

The expression (6.3) is the local version of (5.2).

We now introduce the localized acoustic assumption by postulating the relation

$$
\frac{1}{1+r(x, t)}=1+\frac{p(x, t)-p_{0}}{\rho c^{2}}
$$

which simply is the result (5.5) localized to the point $(x, t)$. It assumes that the static sound speed $c$ and the pressure $p_{0}$ are constants. 
An additional evolution equation can be obtained by differentiating (6.4) with respect to time. In order to obtain a suitable expression we need to find the time derivative of $r$ by use of the identity (6.3). For this purpose we use Euler's formula [15, p. 131] in Lagrangian form. The result is

$$
\partial_{t} r(x, t)=\partial_{t} J(x, t)=J \nabla_{X} \cdot V(X, t)=J(x, t) L_{x} \psi(x, t)^{-T}: L_{x} v(x, t)=(1+r) L_{x} \psi(x, t)^{-T}: L_{x} v(x, t) .
$$

We have of course made use of the formula (2.2). Now differentiation of (6.4) and simplification with the aid of (6.5) yields the evolution equation

$$
\partial_{t} p(x, t)+\left[\rho c^{2}-p_{0}+p(x, t)\right] L_{x} \psi(x, t)^{-T}: L_{x} v(x, t)=0,
$$

which augments the momentum equation (4.4).

When the acoustic assumption is combined with (4.4), the determinant $J$ is eliminated and the equation

$$
\partial_{t} v(x, t)+\left[\frac{c^{2}}{\rho c^{2}-p_{0}+p(x, t)}\right] L_{x} \psi(x, t)^{-T} \nabla_{x} p(x, t)=0
$$

is the result.

The considerations of this section also lead to a constraint. Since the Jacobian $J$ is positive, it follows from (6.2) that $1+r>0$ which means that there is a lower bound to the compression experienced at any given point. A gas cannot be compressed to an arbitrarily small volume. In the light of the acoustic assumption (6.4) there is also a lower bound on the pressures that can develop, namely

$$
1+\frac{p(x, t)-p_{0}}{\rho c^{2}}>0
$$

It should be noticed that, since $\rho c^{2}$ has the unit of pressure, this makes sense.

\section{Scaling}

In equations (6.6) and (6.7) the parameters $\rho$ and $c$ occur. If the reference density $\rho$ is constant, these equations can be scaled into dimensionless form in such a way that the parameters are there no longer. In addition the pressure $p_{0}$ which gives rise to the totally relaxed state $r=0$, can simply be absorbed in the pressure term and therefore ignored.

For the purpose of scaling we assume that there is some length parameter $L$ in terms of which position will be scaled. Time is then scaled in terms of $T=L / c$, velocity in terms of $c$ and pressure in terms of $\rho c^{2}$. Thus the substitutions $x \rightarrow x / L ; t \rightarrow t / T ; v \rightarrow v / c ; p \rightarrow p /\left(\rho c^{2}\right)$ lead to the equations

$$
\begin{aligned}
& v_{t}(x, t)+[1+p(x, t)]^{-1} L_{x} \psi(x, t)^{-T} \nabla_{x} p(x, t)=0 ; \\
& p_{t}(x, t)+[1+p(x, t)] L_{x} \psi(x, t)^{-T}: L_{x} v(x, t)=0 .
\end{aligned}
$$

In addition the constraint (6.8) now takes the form

$$
1+p(x, t)>0 \text {. }
$$

The scaled form of the acoustic assumption (6.4) is

$$
[1+p(x, t)][1+r(x, t)]=1,
$$

which is analogous to the Boyle-Marriott law.

In the sequel we shall work with these equations, but they are still insufficient in number since the three components of the motion $\psi$ are also unknown. From the system (7.1) and (7.2) of transport equations it is seen that there are propagation speeds which depend on pressure in a very specific way. This is in accord with the early intuition of Earnshaw [4,5]. In the remaining sections we study a particular form of the motion in which the problem of the determination of the motion itself is remedied.

\section{One-dimensional motion}

The original studies of the propagation of sound, see e.g. [17,1], were very much focused on one-dimensional motions. Let us therefore consider motions of the form $\psi(x, t)=\left(\psi\left(x_{1}, t\right), x_{2}, x_{3}\right)$. For this motion $L_{x} \psi(x, t)=\operatorname{diag}\left(\partial_{1} \psi, 1,1\right)$ and therefore $J(x, t)=\partial_{1} \psi\left(x_{1}, t\right)$. It is seen that $v(x, t)=\left(\partial_{t} \psi\left(x_{1}, t\right), 0,0\right)$ and, by $(7.4), 1+r(x, t)=\partial_{1} \psi=(1+p(x, t))^{-1}$. Moreover, $L_{x} \psi(x, t)^{-T}=\left[\left(\partial_{1} \psi\right)\right]^{-1} \operatorname{diag}\left(1, \partial_{1} \psi, \partial_{1} \psi\right)=(1+p) \operatorname{diag}\left(1,(1+p)^{-1},(1+p)^{-1}\right)$ and $L_{x} v(x, t)=\operatorname{diag}\left(\partial_{1} v_{1}, 0,0\right)$. The notation 'diag' represents a diagonal matrices. Equations (7.1) now have the form

$$
\begin{aligned}
& \partial_{t} v_{1}\left(x_{1}, t\right)+\partial_{1} p(x, t)=0 \\
& (1+r(x, t)) \partial_{2} p(x, t)=0 \\
& (1+r(x, t)) \partial_{3} p(x, t)=0
\end{aligned}
$$

It follows that $p(x, t)=p\left(x_{1}, t\right)$. 
Equation (7.2) becomes

$$
\partial_{t} p\left(x_{1}, t\right)+[1+p(x, t)]^{2} \partial_{1} v_{1}=0 .
$$

For the one-dimensional case the notation can be simplified by writing $x$ for $x_{1}$ and $v$ for $v_{1}$. Also, in suitable notation for partial derivatives, Equations (8.1) and (8.2) are now written in the form

$$
\begin{aligned}
& v_{t}(x, t)+p_{x}(x, t)=0 \\
& p_{t}(x, t)+[1+p(x, t)]^{2} v_{x}(x, t)=0
\end{aligned}
$$

The assumption of one-dimensionality of the motion therefore resolved the problem of finding more equations in order to determine the motion $\psi$. Indeed, once equations (8.3) and (8.4) are solved, the motion can be determined from the expressions found for $\psi_{x}$ and $\psi_{t}$. The equations we have found are essentially nonlinear-a point that will become even clearer as we go on.

\section{Linearization}

When it is assumed that the compression is 'small' $(|r| \ll 1)$, it follows from (7.4) that the same is true for the pressure. This leads to the following linearization of the system (8.3) and (8.4):

$$
\begin{aligned}
& v_{t}(x, t)+p_{x}(x, t)=0 \\
& p_{t}(x, t)+v_{x}(x, t)=0 .
\end{aligned}
$$

This system leads to the wave equation through elimination of either $v$ or $p$ by taking suitable derivatives. As was pointed out in $[7,8]$, it is unnecessary to go to so much trouble. If we let $u_{1}:=[v+p] / 2$ and $u_{2}:=[v-p] / 2$, addition and subtraction of the two linear equations (9.1) and (9.2) lead to the decoupled equations

$$
\begin{aligned}
& u_{1, t}(x, t)+u_{1, x}(x, t)=0 \\
& u_{2, t}(x, t)-u_{2, x}(x, t)=0
\end{aligned}
$$

For $-\infty<x<\infty$ and $t>0$ the Cauchy problem (9.3) and (9.4) to which the initial conditions

$$
\left.\begin{array}{l}
u_{1}(x, 0)=u_{10}(x) ; \\
u_{2}(x, 0)=u_{20}(x),
\end{array}\right\}
$$

are added, is readily solved by the method of characteristics. The solutions are

$$
\left.\begin{array}{l}
u_{1}(x, t)=u_{10}(x-t) \\
u_{2}(x, t)=u_{20}(x+t) .
\end{array}\right\}
$$

It is quite simple to obtain the initial states $u_{10}, u_{20}$ from initial states of $v$ and $p$ and equally simple to translate from the solutions $u_{1}, u_{2}$ back to $v$ and $p$. Thus the linearized equations pose no formal difficulties and the solutions obtained are typical 'sound waves' in the traditional sense. The only difficulty would be obedience to the constraint (7.3).

\section{How nonlinear is nonlinear?}

Comparison of the nonlinear system (8.3) and (8.4) with the linearized version (9.1) and (9.2) shows the following: only the second equation in the nonlinear system is nonlinear; in the linearized system the roles of $v$ and $p$ can be interchanged which does not happen in the nonlinear system. Furthermore, the decoupling trick that worked for the linearized system does not work for the nonlinear system. As was remarked before, the linearized equations do not depend on the constraint (7.3) while the whole character of the nonlinear system is changed if there were points where $1+p(x, t)=0$. As such points the system is no longer hyperbolic $[2,3,9]$ and the wave-like behaviour of solutions may be at risk.

Let us incorporate the constraint into the equations by making the substitution

$$
1+p(x, t)=\exp \{q(x, t)\} .
$$

Equations (8.3) and (8.4) now become

$$
\left.\begin{array}{l}
v_{t}(x, t)+\exp \{q(x, t)\} q_{x}(x, t)=0 \\
q_{t}(x, t)+\exp \{q(x, t)\} v_{x}(x, t)=0 .
\end{array}\right\}
$$

The transformed system (10.2) has gained a remarkable symmetry even though the new equations are both nonlinear. The other advantage is that the substitutions

$$
\left.\begin{array}{l}
u_{1}(x, t)=\frac{1}{2}[v(x, t)+q(x, t)] ; \\
u_{2}(x, t)=\frac{1}{2}[v(x, t)-q(x, t)]
\end{array}\right\}
$$


which worked so well in the linear system, at least have some effect although the system is not decoupled. The resulting equations are

$$
\left.\begin{array}{l}
u_{1, t}(x, t)+\exp \{q(x, t)\} u_{1, x}(x, t)=0 ; \\
u_{2, t}(x, t)-\exp \{q(x, t)\} u_{2, x}(x, t)=0 \\
q(x, t)=u_{1}(x, t)-u_{2}(x, t)
\end{array}\right\}
$$

Still, the roles of $u_{1}$ and $u_{2}$ cannot be switched. These are the equations we shall use to analyze the nonlinear acoustic problem.

\section{Characteristics of the system}

The system (10.4) has two families of characteristic curves $C_{+}: x=\Xi_{+}(t)$ and $C_{-}: x=\Xi_{-}(t)$ determined by the ordinary differential equations

$$
\left.\begin{array}{l}
\Xi_{+}^{\prime}(t)=\exp \left\{q_{+}(t)\right\} \\
\Xi_{-}^{\prime}(t)=-\exp \left\{q_{-}(t)\right\}
\end{array}\right\}
$$

with $q_{+}$and $q_{-}$the evaluations of $q$ on $C_{+}$and $C_{-}$, respectively. On $C_{+}, u_{1}$ is constant while $u_{2}$ is constant along $C_{-}$. It is seen from (11.1) that $C_{+}$is an increasing curve while $C_{-}$is decreasing.

We shall be concerned with the initial value problem

$$
\left.\begin{array}{l}
u_{1}(x, 0)=u_{10}(x) ; \\
u_{2}(x, 0)=u_{20}(x) ;
\end{array}\right\}
$$

for the case $-\infty<x<\infty$. In this situation, if $\Xi_{+}(0)=\alpha$ and $\Xi_{-}(0)=\beta$,

$$
\left.\begin{array}{l}
q_{+}(t)=u_{10}(\alpha)-u_{2}\left(\Xi_{+}(t), t\right) ; \\
q_{-}(t)=u_{1}\left(\Xi_{-}(t), t\right)-u_{20}(\beta) .
\end{array}\right\}
$$

It becomes clear from (11.1) and (11.3) that the characteristics depend on the initial values prescribed in (11.2) and that the calculation of the characteristics is close to a catch-22 situation. This is alleviated by following the analysis presented in [2] (see also [9]).

We introduce a coordinate system with variables $\alpha, \beta$ which in the $x-t$ plane coincides with the characteristics $C_{+}, C_{-}$. That is, $\alpha=$ const corresponds to a $C_{+}$-curve and $\beta=$ const to a $C_{-}$-curve. In such a coordinate system points in the $x-t$ plane are related to points in the $\alpha-\beta$ plane by $x=X(\alpha, \beta) ; t=T(\alpha, \beta)$. Equations (11.3) now transform into

$$
\begin{aligned}
& X_{\beta}(\alpha, \beta)=\exp \left\{u_{10}(\alpha)-u_{20}(\beta)\right\} T_{\beta}(\alpha, \beta) \text { along } C_{+} \\
& X_{\alpha}(\alpha, \beta)=-\exp \left\{u_{10}(\alpha)-u_{20}(\beta)\right\} T_{\alpha}(\alpha, \beta) \text { along } C_{-} .
\end{aligned}
$$

The initial manifold $t=0$ translates to the line $\beta=\alpha$ and the boundary conditions to go along with Equations (11.4) and (11.5) are

$$
\left.\begin{array}{l}
X(\alpha, \alpha)=\alpha \\
T(\alpha, \alpha)=0 .
\end{array}\right\}
$$

If we follow [2] the next step would be to eliminate $X$ from equations (11.4) and (11.5) to obtain a second order partial differential equation for $T$. We shall refrain from this and rather obtain an integral equation for $T$ which turns out to be handy in the subsequent numerical calculations. For this purpose we re-write the equations in the form:

$$
\begin{aligned}
& \partial_{\beta}\left[X(\alpha, \beta)-\exp \left\{u_{10}(\alpha)-u_{20}(\beta)\right\} T(\alpha, \beta)\right]=\exp \left\{u_{10}(\alpha)-u_{20}(\beta)\right\} u_{20}^{\prime}(\beta) T(\alpha, \beta) ; \\
& \partial_{\alpha}\left[X(\alpha, \beta)+\exp \left\{u_{10}(\alpha)-u_{20}(\beta)\right\} T(\alpha, \beta)\right]=\exp \left\{u_{10}(\alpha)-u_{20}(\beta)\right\} u_{10}^{\prime}(\alpha) T(\alpha, \beta) .
\end{aligned}
$$

Integration of the expressions (11.7) and (11.8) and taking into account the boundary conditions (11.6) yields:

$$
\begin{aligned}
& X(\alpha, \beta)-\exp \left\{u_{10}(\alpha)-u_{20}(\beta)\right\} T(\alpha, \beta)=\alpha+\int_{\alpha}^{\beta} \exp \left\{u_{10}(\alpha)-u_{20}(\eta)\right\} u_{20}^{\prime}(\eta) T(\alpha, \eta) d \eta \\
& X(\alpha, \beta)+\exp \left\{u_{10}(\alpha)-u_{20}(\beta)\right\} T(\alpha, \beta)=\beta-\int_{\alpha}^{\beta} \exp \left\{u_{10}(\theta)-u_{20}(\beta)\right\} u_{10}^{\prime}(\theta) T(\theta, \beta) d \theta .
\end{aligned}
$$

Here we use the variable $\eta$ to indicate that the integration is along a line $\alpha=$ const and $\theta$ for integration along $\beta=$ const. Subtraction of (11.9) and (11.10) yields the integral equation 


$$
\begin{aligned}
& 2 \exp \left\{u_{10}(\alpha)-u_{20}(\beta)\right\} T(\alpha, \beta)=\beta-\alpha-\int_{\alpha}^{\beta} \exp \left\{u_{10}(\theta)-u_{20}(\beta)\right\} u_{10}^{\prime}(\theta) T(\theta, \beta) d \theta \\
& -\int_{\alpha}^{\beta} \exp \left\{u_{10}(\alpha)-u_{20}(\eta)\right\} u_{20}^{\prime}(\eta) T(\alpha, \eta) d \eta
\end{aligned}
$$

\section{Computation of characteristics}

In this section we describe how the characteristics $C_{+}$emanating from the point $(\alpha, 0)$ in the $x$ - $t$ plane and $C_{-}$emanating from the point $(\beta, 0), \alpha<\beta$, may be computed with the integral equation (11.11) as basis. In the $\alpha-\beta$ plane the two points in question are $(\alpha, \alpha)$ and $(\beta, \beta)$ on the 'initial manifold' $\beta=\alpha$. A computational grid is set up by partitioning the interval $[\alpha, \beta]$ into equal sub-intervals of length $h=(\beta-\alpha) / N$. This gives rise to a grid of rectangles as shown in Fig. 1 .

Let $\eta_{k}:=\alpha+k h$ and $\theta_{k}:=\alpha+k h ; k=1, \ldots, N$. Since the computations will recursively cover the 'diagonal' points denoted as 'Levels' (Fig. 1) we need to index the points on the grid accordingly. Thus the grid point $(n, k)$ will refer to the point on Level $n$ with coordinates $\left(\theta_{k}, \eta_{k+n}\right) ; 0 \leqslant n \leqslant N ; 0 \leqslant k \leqslant N-n$. The following indexed notation will be used in accordance: $T_{n, k}, X_{n, k}$ for the values of $T$ and $X$ at the grid point $(n, k)$. In reference to the integrals in (11.11) we define the functions $f_{1}, f_{2}$ as follows:

$$
\left.\begin{array}{l}
f_{1}(\theta):=\exp \left\{u_{10}(\theta)\right\} u_{10}^{\prime}(\theta) \\
f_{2}(\eta):=\exp \left\{-u_{20}(\eta)\right\} u_{20}^{\prime}(\eta) .
\end{array}\right\}
$$

At grid points we shall use the notation $f_{1, k}:=f_{1}\left(\theta_{k}\right), f_{2, k}:=f_{2}\left(\eta_{k}\right)$.

From Fig. 1 it is seen that in order to compute $T(\alpha, \beta)$ all the $T$-values in the triangle below and right of $(\alpha, \beta)$ should be known. To do this we propose an algorithm in which $T$-values at those grid points are computed recursively, starting at Level 0 to obtain the values at Level 1 ; and so on.

The crux of the numerical method is to approximate the integrals in (11.11) by the trapezium-rule. The first step is to go from Level 0 , where, by (11.6), $T_{0, k}=0$. We wish to compute the $T$-values at Level $1, i . e ., T_{1, k}=T\left(\theta_{k}, \eta_{k+1}\right) ; k=0, \ldots, N-1$. The partition of the interval $(\alpha, \beta)$ remains the same throughout. At this transition the integrals in $(11.11)$ are with $\alpha=\theta_{k}$ to $\beta=\eta_{k+1}$. The result is

$$
\begin{aligned}
& \int_{\theta_{k}}^{\eta_{k+1}} \exp \left\{u_{10}(\theta)\right\} u_{10}^{\prime}(\theta) T\left(\theta, \eta_{k+1}\right) d \theta=\int_{\theta_{k}}^{\eta_{k+1}} f_{1}(\theta) T\left(\theta, \eta_{k+1}\right) d \theta \approx \frac{h}{2} f_{1}\left(\theta_{k}\right) T\left(\theta_{k}, \eta_{k+1}\right)=\frac{h}{2} f_{1, k} T_{1, k} \\
& \int_{\theta_{k}}^{\eta_{k+1}} \exp \left\{-u_{20}(\eta)\right\} u_{20}^{\prime}(\eta) T\left(\theta_{k}, \eta\right) d \eta=\int_{\theta_{k}}^{\eta_{k+1}} f_{2}(\eta) T\left(\theta_{k}, \eta\right) d \eta \approx \frac{h}{2} f_{2}\left(\eta_{k+1}\right) T\left(\theta_{k}, \eta_{k+1}\right)=\frac{h}{2} f_{2, k+1} T_{1, k} .
\end{aligned}
$$

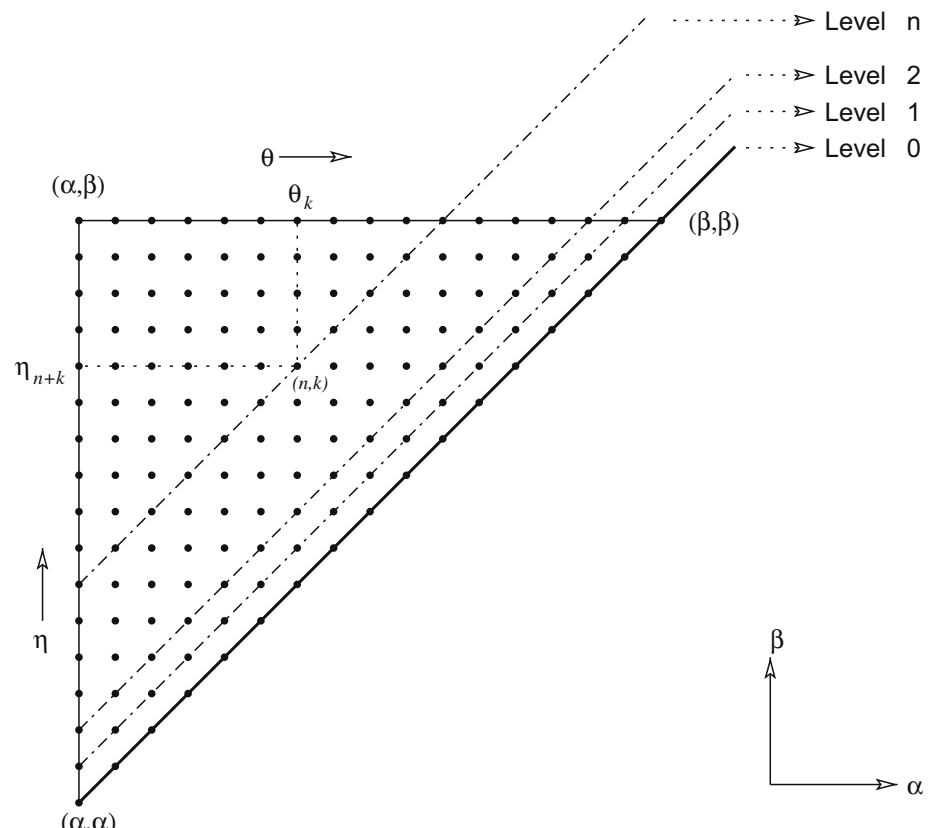

Fig. 1. Computational scheme. 
If the expressions (12.2a) and (12.2b) together with (12.1) are substituted into (11.11) we obtain, after some re-arrangement, the approximate equation

$$
\exp \left\{u_{10}\left(\theta_{k}\right)-u_{20}\left(\eta_{k+1}\right)\right\}\left[2+\frac{h}{2}\left\{u_{10}^{\prime}\left(\theta_{k}\right)+u_{20}^{\prime}\left(\eta_{k+1}\right)\right\}\right] T_{1, k}=\eta_{k+1}-\theta_{k}=h .
$$

Once $T_{1, k}$ is computed from (12.3), it can be substituted into (11.9) to solve for $X_{1, k}(0 \leqslant k \leqslant N-1)$. At this stage an approximate value for the integral is also available.

Next we describe the algorithm for moving from Level $(n-1)$ to Level $n$. That is, $T_{j, k}$ have been calculated for $1<j \leqslant n-1$ and $T_{n, k} ; 0 \leqslant k \leqslant N-n$ must be found. For such $k$ we take $\alpha=\theta_{k}$ and $\beta=\eta_{k+n}$ in (11.11). Now the trapezium-rule involves more than just two grid points and the integrals in (11.11) are approximated as follows:

$$
\begin{aligned}
\int_{\theta_{k}}^{\eta_{k+n}} \exp \left\{u_{10}(\theta)\right\} u_{10}^{\prime}(\theta) T\left(\theta, \eta_{k+n}\right) d \theta & =\int_{\theta_{k}}^{\eta_{k+n}} f_{1}(\theta) T\left(\theta, \eta_{k+n}\right) d \theta \approx \frac{h}{2} f_{1}\left(\theta_{k}\right) T\left(\theta_{k}, \eta_{k+n}\right)+h \sum_{j=1}^{n-1} f_{1}\left(\theta_{k+j}\right) T\left(\theta_{k+j}, \eta_{k+n}\right) \\
& =\frac{h}{2} f_{1, k} T_{n, k}+h \sum_{j=1}^{n-1} f_{1, k+j} T_{n-j, k+j} ; \\
\int_{\theta_{k}}^{\eta_{k+n}} \exp \left\{-u_{20}(\eta)\right\} u_{20}^{\prime}(\eta) T\left(\theta_{k}, \eta\right) d \eta & =\int_{\theta_{k}}^{\eta_{k+n}} f_{2}(\eta) T\left(\theta_{k}, \eta\right) d \eta \approx \frac{h}{2} f_{2}\left(\eta_{k+n}\right) T\left(\theta_{k}, \eta_{k+n}\right)+h \sum_{j=1}^{n-1} f_{2}\left(\eta_{k+j}\right) T\left(\theta_{k}, \eta_{k+j}\right) \\
& =\frac{h}{2} f_{2, k+n} T_{n, k}+h \sum_{j=1}^{n-1} f_{2, k+j} T_{j, k} .
\end{aligned}
$$

When equations (12.4a) and (12.4b) are fed back into the general expressions we obtain

$$
\begin{aligned}
& \exp \left\{u_{10}\left(\theta_{k}\right)-u_{20}\left(\eta_{k+n}\right)\right\}\left[2+\frac{h}{2}\left\{u_{10}^{\prime}\left(\theta_{k}\right)+u_{20}^{\prime}\left(\eta_{k+n}\right)\right\}\right] T_{n, k} \\
& =n h-h \exp \left\{-u_{20}\left(\eta_{k+n}\right)\right\} \sum_{j=1}^{n-1} f_{1, k+j} T_{n-j, k+j}-h \exp \left\{u_{10}\left(\theta_{k}\right)\right\} \sum_{j=1}^{n-1} f_{2, k+j} T_{j, k} .
\end{aligned}
$$

Again, if $T_{n, k}$ is computed from (12.5), $X_{n, k}$ can be obtained from an approximate form of (11.9). The recursion is followed up to Level $N$. It should be noted that in the process a number of in-between characteristics are obtained as well. In Fig. 2 we show some of the $C_{+}$characteristics associated with the initial conditions $u_{10}(x)=u_{20}(x)=\exp \left\{-x^{2}\right\} / 2$ which correspond to the physical initial conditions $v(x, 0)=\exp \left\{-x^{2}\right\}, p(x, 0)=0$.

It is seen that the characteristics intersect which means that 'shock discontinuities' form after a finite time. This is due to a decreasing part of the initial state. For a single nonlinear transport equation this phenomenon is beautifully explained in [12]. For a system the situation is more complex [10] and will be discussed in a forthcoming paper. It should be noted that in the linear case (Section 9) where the characteristics are parallel lines, this cannot occur.

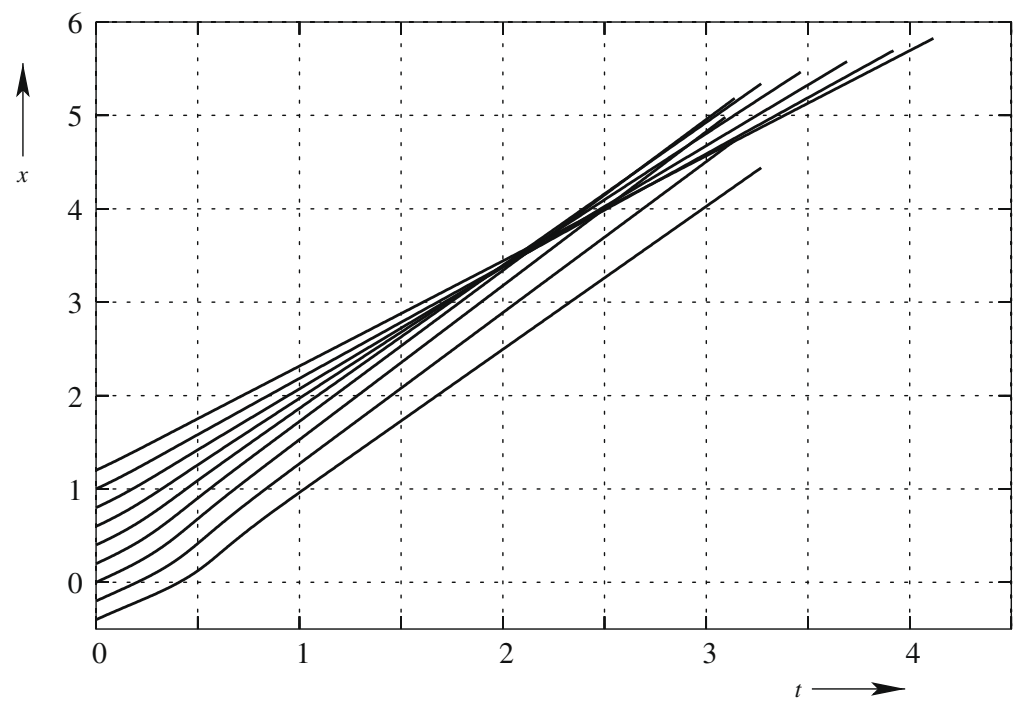

Fig. 2. Increasing characteristics. 


\section{The domain of dependence problem}

The question of existence and uniqueness of a quasilinear system such as (10.4) has been adequately settled in [6,11]. To be able to compute solution curves for the initial value problem at a given time $t$ it is necessary to find the domain of dependence $[\alpha, \beta]$ of a given point $(x, t)$. That is, to find $\alpha$ such that the characteristic $\mathrm{C}_{+}$passes through $(\alpha, 0)$ and $(x, t)$ as well as $\beta$ such that the $C_{-}$passes through $(\beta, 0)$ and $(x, t)$.

A reasonable way of doing this is to obtain a lower bound for $\alpha$ and an upper bound for $\beta$. For this purpose we integrate equation (11.1) and use the integral mean value theorem to obtain

$$
\begin{aligned}
& x=\alpha+\exp \left\{u_{10}(\alpha)\right\} \int_{0}^{t} \exp \left\{u_{2}\left(\Xi_{+}(\sigma), \sigma\right)\right\} d \sigma=\alpha+t \exp \left\{-u_{10}(\alpha)\right\} \exp \left\{u_{2}\left(\Xi_{+}(\eta), \eta\right)\right\} \\
& x=\beta+\exp \left\{-u_{20}(\beta)\right\} \int_{0}^{t} \exp \left\{u_{1}\left(\Xi_{-}(\sigma), \sigma\right)\right\} d \sigma=\beta+t \exp \left\{-u_{20}(\beta)\right\} \exp \left\{u_{1}\left(\Xi_{-}(\theta), \theta\right)\right\}
\end{aligned}
$$

A quick mental sketch of the characteristics involved leads us to realize that $u_{2}\left(\Xi_{+}(\eta), \eta\right)=u_{20}\left(\eta^{*}\right)$ and that $u_{1}\left(\Xi_{-}(\theta), \theta\right)=u_{10}\left(\theta^{*}\right)$ for some $\eta^{*}, \theta^{*} \in[\alpha, \beta]$. Thus (13.1a) and (13.1b) lead to

$$
\left.\begin{array}{l}
x=\alpha+t \exp \left\{u_{10}(\alpha)\right\} \exp \left\{-u_{20}\left(\eta^{*}\right)\right\} \\
x=\beta-t \exp \left\{-u_{20}(\beta)\right\} \exp \left\{u_{10}\left(\theta^{*}\right)\right\} .
\end{array}\right\}
$$

If $M_{2}:=\sup \exp \left\{-u_{20}\left(\eta^{*}\right)\right\}$ and $M_{1}:=\sup \exp \left\{u_{10}\left(\theta^{*}\right)\right\}$ then (13.2) gives rise to the inequalities

$$
\left.\begin{array}{l}
\alpha \geqslant x-M_{2} \exp \left\{u_{10}(\alpha)\right\} t \\
\beta \leqslant x+M_{1} \exp \left\{-u_{20}(\beta)\right\} t .
\end{array}\right\}
$$

The best lower and upper bounds under this approach are given by the solutions of (13.3) with equality signs.

The way to go about the computation of solution curves for fixed $t$ is to begin with the lower bound for $\alpha$ and the upper bound for $\beta$ as described above and to use the method of Section 12 to calculate the $x$ and $t$-values associated with these. Since we start with lower and upper bounds, the $t$-value will be too large. It can be diminished in a systematic way to approach the given $t$-value. The process can be accelerated by use of the old and trusted regula falsi method to come within any given degree of accuracy. The $x$-value obtained, is not of much consequence. In the end, running through a sequence of $x$-inputs and recording the associated $x$-outputs, yields a good graphical representation of the evolution of a given input. In Fig. 3 a few of the computed velocity profiles which evolve from the initial states $v_{0}(x):=v(x, 0)=0.5 \exp \left\{-x^{2}\right\}, p_{0}$ : $=p(x, 0)=0$ are displayed. In this situation (10.3) shows that $u_{10}(x)=u_{20}(x)=v_{0}(x) / 2$. The time frames involved are $t=0.1 ; 0.5 ; 1.0 ; 2.0 ; 3.0$. The 'warping' effect of the impending shock discontinuity can be observed. The effect of the initial inputs on pressure can also be calculated. This is shown in Fig. 4. Once again the approaching shock can be seen.

Apart from the deforming effect due to decreasing portions of the initial velocity, there is a qualitative similarity with the linear theory in the sense that the velocity profile divides into two halves, the one moving downstream and the other upstream. In the pressure profiles the up and downstream parts are of opposite 'phases' similar to the linear case where the solutions of (9.3)-(9.5) are given by (9.6).

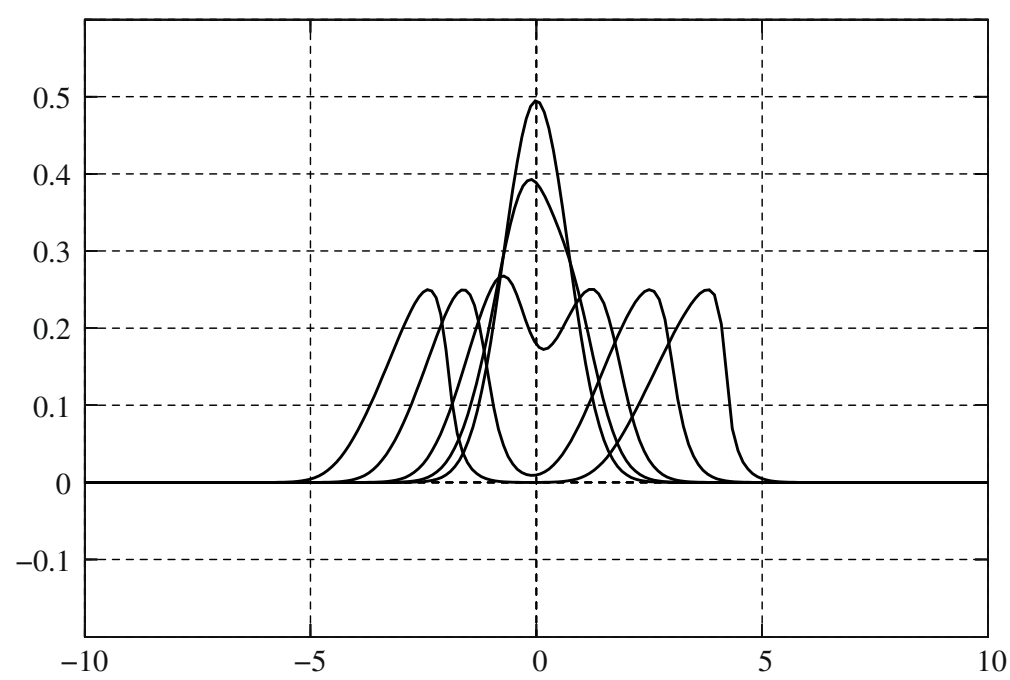

Fig. 3. Computed velocity profiles; zero initial pressure. 


\section{The switching of roles}

Here we briefly indicate the effect of switching to zero initial velocity in contrast to the computations of the previous section. According to $(10.1) q_{0}(x)=\ln \left\{1+p_{0}(x)\right\}$. By (10.3) the initial states that go into the computation will be $u_{10}(x)=q_{0}(x) / 2=\ln \sqrt{1+p_{0}(x)}=-u_{20}(x)$. This is quite different from the situation in Section 13 and the results are bound to be different. For the initial state $p_{0}(x)=0.5 \exp \left\{=x^{2}\right\}$ the results are shown in Figs. 5 and 6 .

The comparisons should be between Figs. 3 and 5 and then between Figs. 4 and 6. It is seen that in Fig. 5 the evolution is much more symmetrical around $x=0$ than in Fig. 3. The same is true when Figs. 4 and 6 are compared. Of interest also is to note that the 'steepening' towards the shock is opposite in the two cases in question. These observations add gravity to the remark in Section 10 that in the basic equations the roles of $v$ and $p$ cannot be reversed and contrast to the linearized equations where they can.

Once again, the effect of looming shock discontinuities can be seen in the skewing of the outgoing disturbances. There seems to be more preservation of symmetry compared to the case when the initial velocity is different from zero.

\section{Pushing against a barrier}

A question that remains is: what happens when the initial input comes close to the constraint (7.3)? The initial state $v_{0}(x)=0,1+p_{0}(x)=A \exp \left\{-x^{2}\right\}=\exp \left\{-\left(a^{2}+x^{2}\right)\right\} ; A=0.5$ was chosen for the computation. Under these conditions

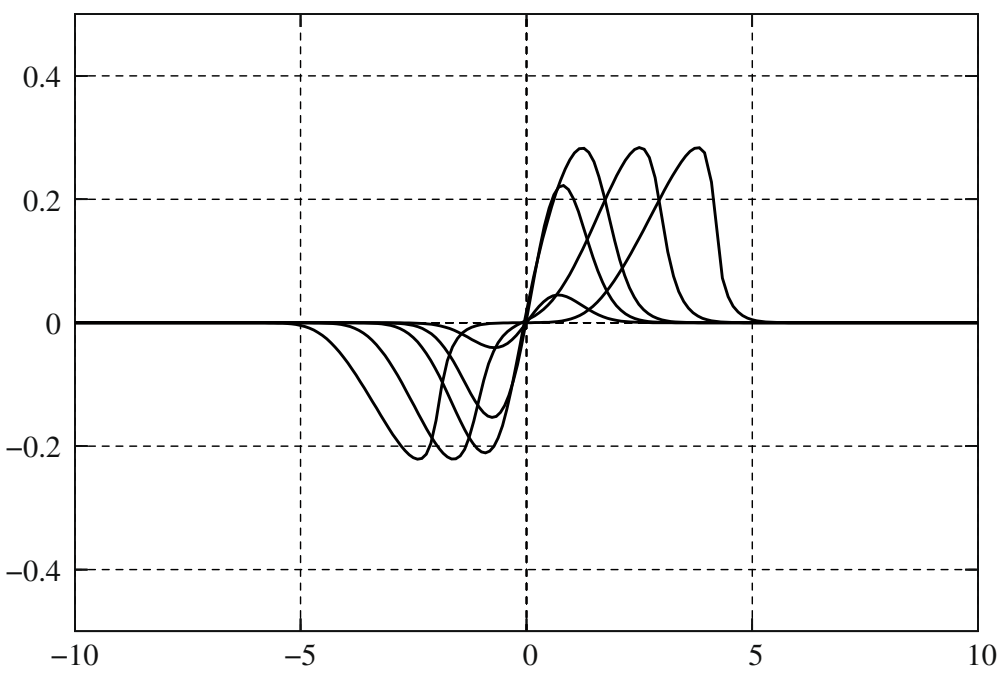

Fig. 4. Computed pressure profiles; zero initial pressure.

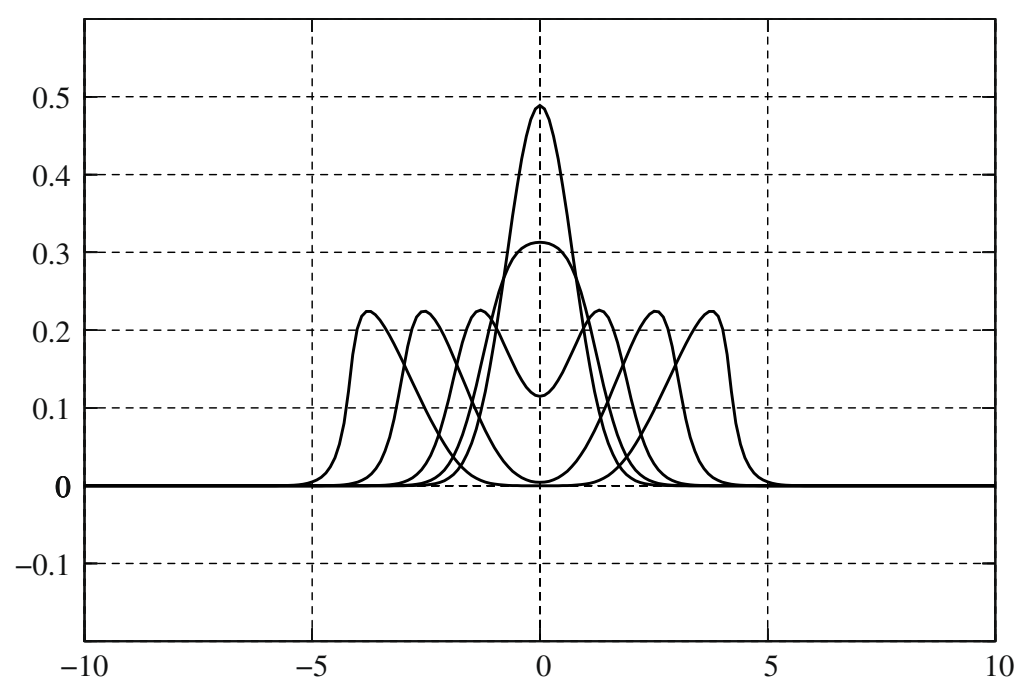

Fig. 5. Computed pressure profiles; zero initial velocity. 


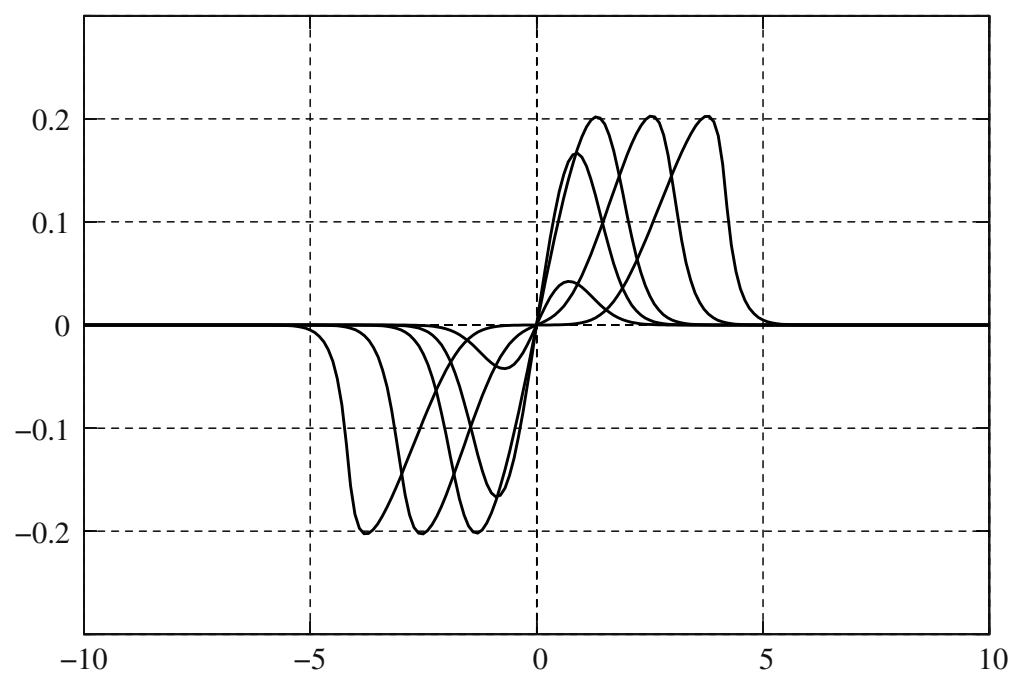

Fig. 6. Computed velocity profiles; zero initial velocity.

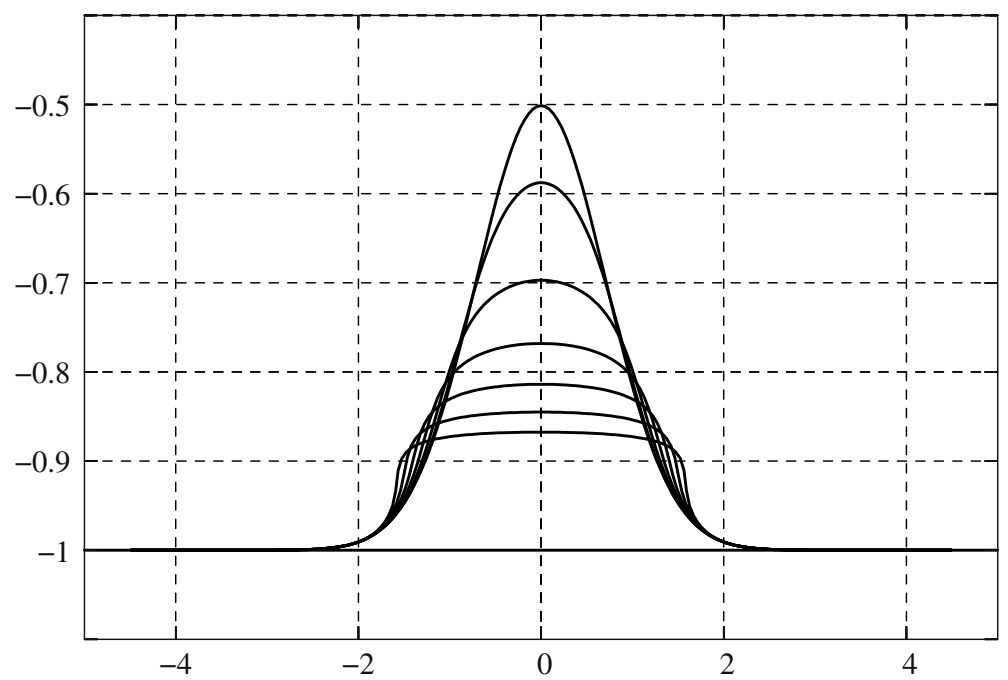

Fig. 7. Development of pressure near the constraint.

$u_{10}(x)=-\left(a^{2}+x^{2}\right) / 2=-u_{20}(x)$. For $x$ 'large' the constraint is initially quite close to being violated, and computationally this is even closer. It is seen that the initial disturbance is not propagated in any wave-like manner. The effect on the development of the pressure under such conditions is shown in Fig. 7. It is seen that the pressure simply sags.

The development of the velocity profiles presents a different picture altogether. The (potential) energy of the initial pressure field is transmuted into kinetic energy 'gone wild'. This is shown in Fig. 8 which is very much reminiscent of the all too well-known empirical curve of the sonic boom, albeit with excess pressure instead of velocity.

\section{The dynamic piston problem}

This work cannot be complete if the piston problem as first suggested in [4,5] is not visited again. A similar problem is addressed outside of acoustics in [2] and, within the framework of water waves, in [16]. The difficulty with these discussions is that a piston is moved from the point $x=0$ (in the Euler description) at a prescribed velocity into a semi-infinite tube. In a more real-to-life situation the motion of such a piston is accomplished by an applied pressure on the piston which is counter-acted by the motion of the gas ahead. In addition the problem is complicated by the Euler description which makes the region occupied by the gas time-dependent. What should be done is to describe the motion of the piston by Newton's second law. 


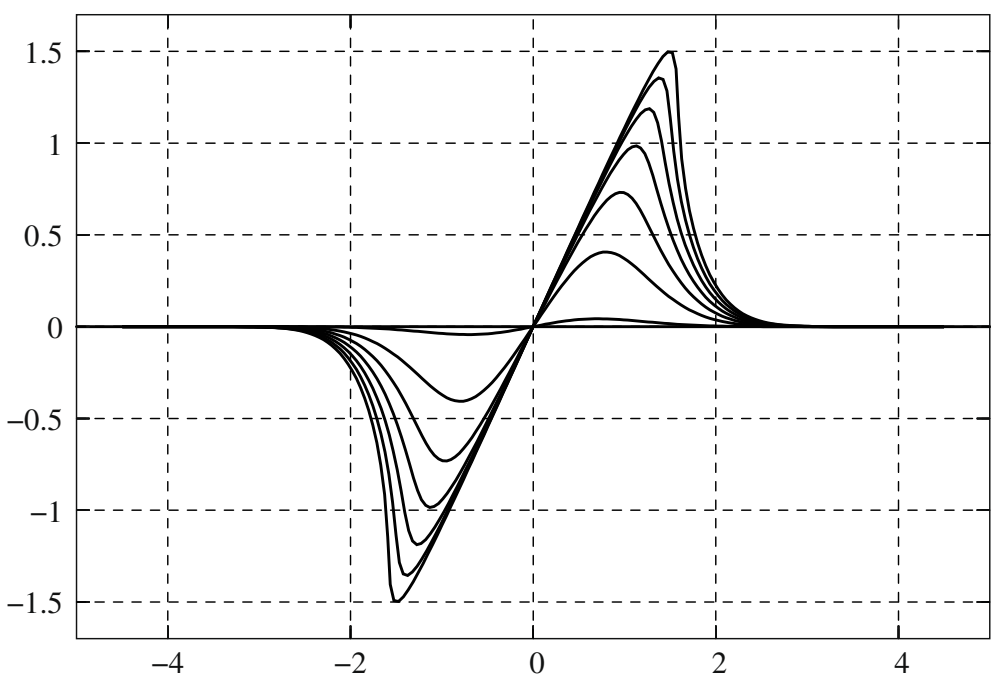

Fig. 8. Development of velocity near the constraint.

In the Lagrangian description, let the reference region be $0<x<\infty$ and let the piston act at $x=0$. Let $M$ denote the mass of the piston, $A$ its cross-sectional area and $V(t)$ its velocity at time $t$. In the notation of Section 4 the equation of motion of the piston is

$$
M V^{\prime}(t)=A P(t)-A p(0, t),
$$

with $P(t)$ the applied pressure. To this we add the contact condition

$$
V(t)=v(0, t)
$$

which states that the particles immediately in front of the piston have the same velocity as the piston. Scaling according to Section 7 and with the choice $L=M /(\rho A)$, equations (16.1) and (16.2) take the form

$$
\frac{d}{d t}[v(0, t)]=P(t)-p(0, t) .
$$

This is a dynamic boundary condition at $x=0$ for the system of equations (8.3) and (8.4). To this we add the 'neutral' initial conditions

$$
\left.\begin{array}{l}
v(x, 0)=0 \\
p(x, 0)=0
\end{array}\right\}
$$

The study of the system of equations (8.3) and (8.4) under the initial conditions (16.4) and the dynamic boundary condition (16.3) is best done by means of the method of characteristics and we re-formulate according to Section 10. From (16.4) we see that $u_{10}(x)=u_{20}(x)=0$ for $x>0$. Hence, from (11.1), the characteristics emanating from the half line $x>0$ are $x=\alpha+t\left(C_{+}\right)$and $x=\beta-t\left(C_{-}\right)$. This implies that $u_{1}(x, t)=0$ in the region $\mathscr{R}=\{0<x<t ; t>0\}$, and that $u_{2}(x, t)=0$ in the entire region $x>0 ; t>0$. The only unknown functions for this problem therefore are $u(x, t):=u_{1}(x, t)$ defined on $\mathscr{R}$ and $w(t):=u_{1}(0, t)$ defined for $t>0$. Now the dynamic boundary condition (16.3) takes the form

$$
w^{\prime}(t)+\exp \{w(t)\}=1+P(t)
$$

having noticed that $1+p(0, t)=\exp \{q(0, t)\}=\exp \{w(t)\}$ from (10.1) and (10.4).

Under the initial condition $w(0)=0$, which is in compliance with (16.4), equation (16.5) can be solved explicitly:

$$
\begin{aligned}
& w(t)=\int_{0}^{t}[1+P(\tau)] d \tau-\ln \left[1+\int_{0}^{t} I(\tau) d \tau\right] \\
& I(t):=\exp \left[\int_{0}^{t}[1+P(\tau)] d \tau\right] .
\end{aligned}
$$

The solution $w(t)$ represents the piston velocity at time $t$. Thus one part of the solution of the problem has been achieved.

For the special case where $P$ is constant, the solution defined in (16.6a) and (16.6b) is even more explicit:

$$
w(t)=(1+P) t+\ln \left[\frac{1+P}{P+\exp \{(1+P) t\}}\right] .
$$


It is seen from (16.7) that if the constant $P$ is positive the solution $w(t)$ is an increasing function of time. It is also seen that for $0>P>-1$ the piston velocity decreases and that for $P=-1$ the expression (16.7) is undefined. It would appear that the constraint (7.3) needs to be obeyed by the applied pressure and that a phenomenon similar to cavitation may be possible (see e.g. [16]). If $P>-1, w(t)$ tends to $\ln (1+P)$ for large times. The 'piston curves' $w(t)$ for positive and negative values of $P$ are shown in Fig. 9. There the fierce resistance to compressive action as opposed to expansion is demonstrated. This is due to the constraint which is present in our equations.

With the motion of the piston known it remains to find the motion of the gas in front of it. The boundary value problem to be solved is, according to the discussion above,

$$
\left.\begin{array}{l}
u_{t}(x, t)+\exp \{u(x, t)\} u_{x}(x, t)=0 ; \quad x>0 ; t>0 ; \\
u(0, t)=w(t) ; \\
u(x, t)=0 \text { for } x>t .
\end{array}\right\}
$$

This is almost a standard problem for single nonlinear transport equations as set out in [12], the complication being the condition in (16.8) at $x=0$. To find the characteristics issuing from that line, it is expedient to represent them as the curves $t=T(x)$. The equation for $T$ then is

$$
T^{\prime}(x)=\exp \{-u(x, T(x))\} .
$$

For the characteristic passing through the point $\left(0, t_{0}\right)$ we have $u(x, T(x))=u(0, T(0))=w\left(t_{0}\right)$ and it follows from (16.9) that its equation is

$$
t=t_{0}+\exp \left\{-w\left(t_{0}\right)\right\} x
$$

An argument similar to that in [12] reveals that if the piston velocity $w(t)$ is increasing at some instant in time the formation of shock discontinuities cannot be avoided. This is certainly the case when the applied pressure $P(t)$ is constant and positive. To compute the value of the solution to (16.8) at a given point $(x, t)$ one has to solve (16.10) for $t_{0}$. Then $u(x, t)=w\left(t_{0}\right)$. For the case of a positive constant applied pressure $P=0.5$ the results are shown in Fig. 10 . One is tempted to refer to the developing discontinuity as Earnshaw's bore.

\section{7. "To arrive where we started ...}

The end of our excursion gives cause for some reflection. From rational principles and analysis we arrived at nonlinear equations (and an inequality) for the propagation of sonic disturbances in an ideal compressible gas. These equations seem to be aligned to the thoughts of Rev. Stephen Earnshaw some 150 years ago. The effects of initial disturbances showed that discontinuities can develop within short time spans and that the roles of velocity and pressure cannot be interchanged. Upon all that, we found that Earnshaw was correct: if gas is forced downstream by an external action a bore-like phenomenon will occur.

When Marco Polo returned to Venice from his extended excursion to the 'far east', his intimations may not immediately have struck new chords. What we have learned is, perhaps, that discoveries may not always be new. Understanding, although newer and fresher, takes longer to mature.

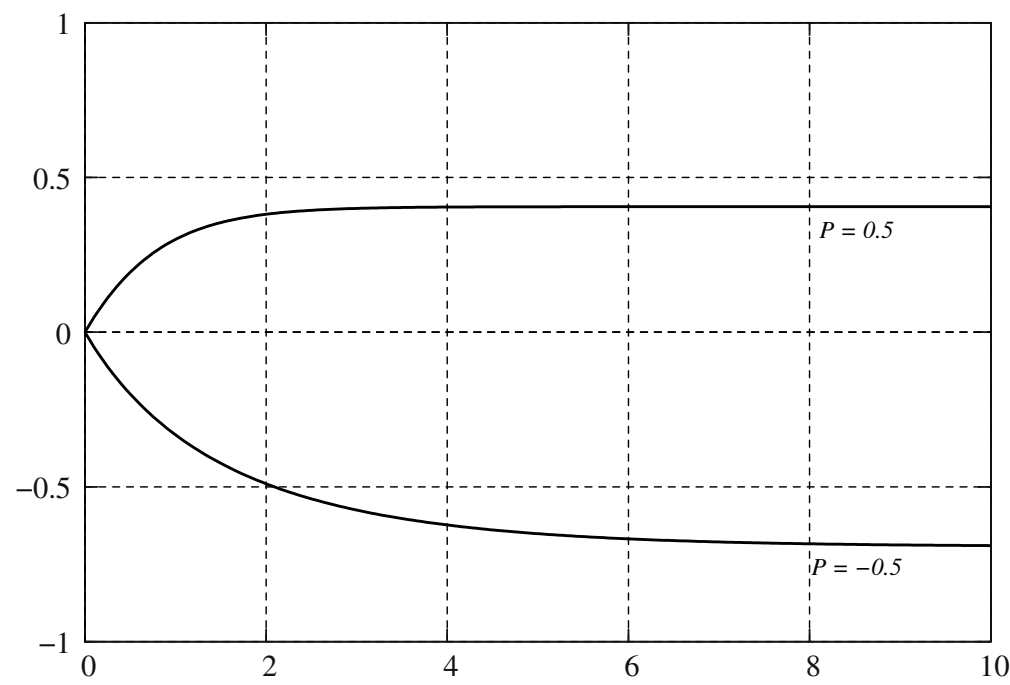

Fig. 9. Piston velocity curves under constant applied pressure. 


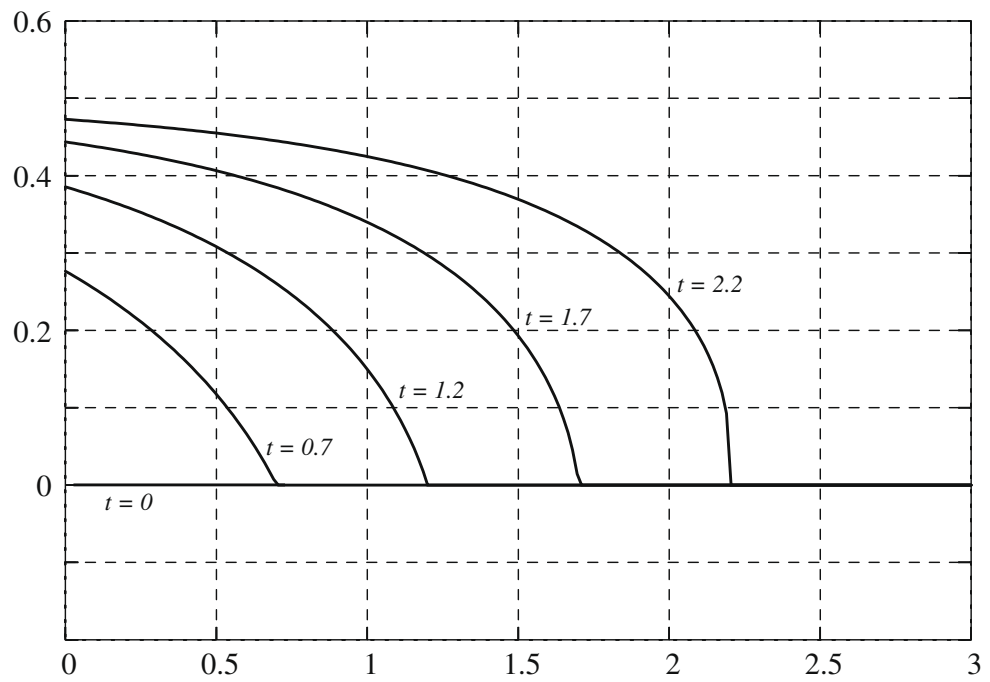

Fig. 10. Velocity profiles due to positive constant applied pressure.

\section{Acknowledgments}

A significant part of the work reported here was done in the relaxed atmosphere of Coffee at Burgundy's on campus where the friendly staff and the aroma of coffee greatly contributed to clear thinking. To my dear friend and colleague Adri Prinsloo who often joined me, a word of sincere appreciation for asking incisive and penetrating non-technical questions (the most difficult of all). They resulted in a deeper understanding of what I was doing and brought us to the mutual conclusion that in the Sciences and the Humanities, the epistemological dilemma is the same.

The figures presented in the text were made with the help of the software packages Gnuplot and Xfig. They attest to the nobility of the idea of Open Source.

\section{References}

[1] C.A. Coulson, Waves, seventh ed., Oliver and Boyd/Interscience Publishers, Edinburgh, London/New York, Reprinted 1961.

[2] R. Courant, K.O. Friedrichs, Supersonic Flow and Shock waves, Interscience Publishers, New York, 1948.

[3] R. Courant, D. Hilbert, Methoden der mathematischen Physik, II, Heidelberger Taschenbücher, vol. 31, Springer-Verlag, Berlin, Heidelberg, New York, 1968.

[4] S. Earnshaw (Rev), On the Mathematical Theory of Sound, Proc. Roy. Soc. London 9 (1857) 590-591.

[5] S. Earnshaw (Rev), On the Mathematical Theory of Sound, Phil. Trans. Roy. Soc. London 150 (1860) 133-148.

[6] K.O. Friedrichs, Nonlinear hyperbolic differential equations for functions of two independent variables, Am. J. Math. 70 (1948) $555-588$.

[7] K.O. Friedrichs, Symmetric hyperbolic linear differential equations, Commun. Pure Appl. Math. 7 (1954) 345-392.

[8] K.O. Friedrichs, Symmetric positive linear differential equations, Commun. Pure Appl. Math. 11 (1958) 333-418.

[9] A. Jeffrey, The evolution of discontinuities in solutions of homogeneous nonlinear hyperbolic equations having smooth initial data, J. Math. Mech. (Indiana J. Math.) 17 (1967) 331-352.

[10] A. Jeffrey, Quasilinear Hyperbolic Systems and Waves, Research Notes in Mathematics, vol. 5, Pitman Publishing, London, San Francisco, Melbourne, 1976.

[11] P.D. Lax, Nonlinear hyperbolic equations, Commun. Pure Appl. Math. 6 (1953) 231-258.

[12] P.D. Lax, The formation and decay of shock waves, Am. Math. Monthly 79 (1972) 227-241.

[13] K.R. Rajagopal, A.R. Srinivasa, On the response of non-dissipative solids, Proc. R. Soc. A. 463 (2007) 357-367.

[14] A. Rozanova-Pierrat, Qualitative Analysis of the Khokhlov-Zabolotskaya-Kuznetsov (KZK) equation, Math. Model. Methods Appl. Sci. 18 (2008) 781812.

[15] J. Serrin, Mathematical principles of classical fluid mechanics, in: S. Flügge (Ed.), Handbuch der Physik, VIII/I, Strömungsmechanik, Springer-Verlag, Berlin, Göttingen, Heidelberg, 1957, pp. 123-263.

[16] J.J. Stoker, Water Waves, Interscience Publishers, New York, 1958.

[17] J.W. Strutt (Baron Rayleigh 3), The Theory of Sound, second ed. revised and enlarged, Dover Publications, New York, 1945. 